\title{
LA IMPLEMENTACIÓN EN EL NIVEL OPERATIVO DEL PROCESO DE ENFERMERÍA
}

\author{
Nora Agnes Vega Villalobos*
}

VILLALOBOS, N.A.V. La implementación en el nivel operativo del proceso de enfermería. Rev.latinoam.enfermagem, Ribeirão Preto, v. 7, n. 1, p. 67-73, janeiro 1999.

El mejoramiento continuo es el marco de referencia utilizado como guía del proceso de enfermería por medio del giro del PDCA. (plan, do, check, act). Traducido en VIDA. (visión, implementación, discernimiento, ajuste). Par aplicarlo se utilizó la teoría de cambio de Kurt Lewin. En este artículo solo se presentan los resultados de la Implementación con las estrategias de cuidado técnico y de educación, administrativo y de investigación. La aplicación de las estrategias demostró que los clientes no manifiestan sus sentimientos, creencias, expectativas ni conquistan su autonomía en el cuidado, tampoco ejercen su ciudadanía para vivir y poder evoluir.

TÉRMINOS CLAVES: implementación, mejoramiento continuo, autonomía, ciudadanía

\section{INTRODUCCION}

El proceso de enfermería es la base del quehacer del profesional enfermero, lo que le permite investigar para poder administrar los cuidados y educar, o sea, construir y participar con el cliente en su cuidado.

Todo enfermero debe poseer un marco de referencia susceptible de cambios que sustenten su actuación en la práctica diaria.

Para efectos de este estudio he formulado como marco de referencia el mejoramiento continuo para el proceso de enfermería en el cuidado pediátrico. El proceso no es una receta pronta que aplico indistintamente a los clientes, sino un compartir, un contrato que debe hacerse de ambos lados para que se pueda ejecutar y evaluar el cuidado que desean y que pueda ser efectivo. Es repensar la asistencia para la mejoría continua de la atención compartida.

El mejoramiento continuo es una estrategia de gestión de calidad. La gestión de calidad es un enfoque de calidad total.

Para que haya gestión de calidad, la investigación es un principio de acción, la administración es necesaria ya que el proceso de atención de enfermería es una forma de administrar ese cuidado. De forma que se pueda profundizar en ese cotidiano de la asistencia y construir una estrategia de trabajo que permita la calidad de atención redireccionando el quehacer del profesional de enfermería (cliente interno) y el contrato establecido con el usuario y su familia.(cliente externo) aplicar el proceso de enfermería con un marco referencial de mejoramiento continuo. El cuidado va a estar determinado por los resultados de la investigación y se dará con la educación, con el diálogo, construyendo y participando.

Mi propósito es demostrar que el proceso de enfermería es lo que me hace ser una profesión independiente, que me permite aplicar todo lo que he estudiado. Me da libertad de escoger el marco de referencia con el que quiero trabajar, según mis creencias. Las técnicas de enfermería me ayudan al acercamiento para influenciar, modificar la imagen de enfermería positiva o negativamente.

Me da satisfacción como enfermera. Es indicar nuevos caminos administrativos, de cuidado, de educación e investigación para el profesional de enfermería en su cotidiano que permita demostrar como la calidad del cuidado es posible a bajo costo y sin deshumanizar el servicio.

Las dificultades probables a encontrar para aplicar el marco de referencia de mejoramiento continuo podrán ser: un profesional de enfermería absorbido por un sistema institucional de trabajo, cansado, desmotivado, con poco estímulo y desconocimiento del marco referencial de mejoría continua. Con un cliente externo acostumbrado o no a un sistema institucional de salud, con poca o mucha expectativa pero sin conocimiento del significado de mejoramiento continuo de la asistencia con el marco referencial que nos proponemos desarrollar. Y un sistema

\footnotetext{
* Master en enfermería y Profesora Asociada de la Escuela de Enfermería de la Universidad de Costa Rica. Doutoranda da Pósgraduação em Enfermagem da U.F.S.C.
} 
institucional que probablemente camina por la ruta de control de calidad pero de difícil acceso a gestión de calidad de conjunto entre cliente interno, externo e institución.

Para obtener el propósito me propuse la siguiente mirada.

\section{MIRADA PROPUESTA}

Desarrollar el proceso de atención que viabilice la calidad del cuidado pediátrico hospitalar por medio de la mejoría continua.

En este artículo solo presentaré los resultados del siguiente objetivo específico:

Establecer las estrategias de "implementación" de cuidado técnico y de educación, administrativas y de investigación para el logro de la mejoría continua.

\section{METODOLOGÍA}

La aplicación del proceso de enfermería se dio en unidades de internamiento de niños con diversos problemas de salud. Mi interés fue el trabajar el nivel operacional de la mejoría continua con los clientes de la institución, o sea el proceso VIDA.

La intervención se realizó aplicando el proceso VIDA. (Visión, Implementación, Discernimiento y Ajuste) para el mejoramiento continuo y las etapas de cambio de Lewin (LEWIN, 1951); (LEDDY \& PEPPER, 1985, p. 316); (CERQUEIRA, 1993). La Visión corresponde al Descongelamiento de Lewin y nos indicará los puntos claves sobre los que se va a actuar o sea la recolección de datos basados en el concepto de la calidad del cuidado del niño. La Implementación y el Discernimiento corresponden a la Reestructuración. y el Ajuste al Recongelamiento. Para efectos de este artículo analizaremos solamente la Implementación.

Las estrategias para la Implementación fueron: la administración, el cuidado, la investigación y la educación.

La administración se consideró cuando se encontraba que el cliente tenía acceso al tratamiento oportuno, se fortalecía la conquista de la autonomía y se redireccionaba el cuidado según las creencias, expectativas y sentimientos de los niños y sus padres.

El Cuidado, cuando se cumplían las órdenes médicas por medio de la aplicación de técnicas de enfermería planeadas o no o cuando se experimentaba lo nuevo para sobrevivir mejor.

La Investigación, cada vez que la familia procura buscar lo mejor del cuidado en el día a día.

La Educación cuando se daba un diálogo para construir nuevas formas de cuidado y participar para vivir a plenitud.

El deseo no era aplicar el proceso de enfermería a niños con determinado diagnóstico clínico, sino el experimentar el uso del referencial de gestión de calidad para el mejoramiento continuo en el cuidado de enfermería pediátrica. Por ello se trabajó con niños con problemas neurológicos, oncológicos, pulmonares, gastrointestinales. Los participantes fueron trece niños de diferentes edades.

\section{MARCO DE ILUMINACIÓN}

El mejoramiento continuo es el nivel operacional del componente de mejoría de gestión de calidad (CERQUEIRA, 1992, p. 11) que hace girar el PDCA (Plan, Do, Check, Act). El Plan es decir y documentar lo que se hace, Do hacer lo que se dice, Check es evaluar lo que se hace, compara los resultados con lo planeado o sea el Momento de la Verdad; y Act es corregir y prevenir las fallas. (CORRÊA, 1993, p. 64).

Para efectos del proceso de enfermería pediátrica el PDCA será entendido como VIDA. Donde $\mathrm{P}=\mathrm{V}$ e indicará la visión o marco de referencia del planeamiento. $\mathrm{La} \mathrm{D}=\mathrm{I}$ o sea las estrategias de acción. $\mathrm{La} \mathrm{C}=\mathrm{D}$ nos dará el Discernimiento del "Momento de la Verdad". Y A=A, demostrará el ajuste del cuidado.

Entenderemos como proceso de enfermería el cambio que implica un cuestionamiento sistemático con una visión que posibilita el intervenir en la práctica con estrategias para obtener calidad en el cuidado en cada Momento de la Verdad y evaluar para poder indicar una nueva visión.

La Visión que corresponde al Descongelamiento estará dada por el concepto de "calidad del cuidado del niño" considerado como aquel que permite que el sistema de referencia del niño, de su familia y del profesional de enfermería (creencias, expectativas, valores, sentimientos, deseos) sean manifiestos y tomados como fundamento en el contexto, en que todos poseen autodeterminación permitiendo que el niño y sus padres soliciten el cuidado cuando lo consideren necesario y que el profesional de enfermería pueda ofrecerlo según la solicitud o su propia proposición. Esa autodeterminación será dada por el ejercicio de la ciudadanía donde el niño y los padres son capaces de elaborar su propio proyecto de calidad de vida, de evolución, de cambio en medio de la enfermedad, de la incerteza para obtener placer y felicidad.

La reestructuración está dada por la Implementación y el Discernimiento. La Implementación está dada por las siguientes estrategias de intervención: administración, cuidado, investigación y educación. Regresaremos a profundizar en ellas ya que son el objeto de nuestro artículo. 
El Discernimiento o Momento de la Verdad lo podemos definir como todo contacto entre el cliente y el profesional de enfermería. Este Momento de la Verdad por sí solo no es positivo ni negativo. La forma en que ese momento es encarado es la que va a transformarlo en positivo o negativo. (ALBRECHT \& BRADFORD, 1992, p. 28).

Después de cada Momento de la Verdad, en la aproximación al cliente y su familia para verificar lo que sucede entramos en la etapa de Ajuste que no es otra cosa que ese filosofar, ese análisis más detallado, ese preguntar constante indispensable para obtener la calidad. Es el recongelamiento momentáneo del cambio.

Ahora, en este marco de referencia volvamos a la Implementación. Aplicando CERQUEIRA (1992, p. 76), el hardware es constituido por la administración y el cuidado (lo que debe ser hecho) y el software (como ser hecho), lo que anima el hardware formado por la investigación y la educación.

Cómo debe darse la calidad del cuidado del niño? Es organizándolo, administrándolo. Administrar es saber enfrentar lo que se quiere que suceda y al mismo tiempo prever lo que está por venir; es lidiar objetiva y eficazmente con las personas de todos los niveles, obtener resultados, tener confianza, aprender a recopilar y a entender informaciones, en resumen ser totalmente participantes (CROSBY, 1993, p. 7). "Administración de la calidad implica un cambio en la cultura de enfermería” (MALIK, 1992, p. 40).

El hospital es el proveedor que será bien sucedido cuando ayude a sus clientes a serlo y estos tendrán que darle ese chance (CROSBY, 1993, p. 55). Un empleado es bien sucedido, tanto en la vida profesional como particular cuando ayuda a los otros a ser bien sucedidos (CROSBY, 1993, p. 27). Si existe algún secreto para tornar al cliente bien sucedido este reside en saber lo que el quiere (CROSBY, 1993, p. 41).

Como ilustración y no como metodología fundamental de este trabajo esa administración representada por Ulises, ese personaje de la mitología griega que fue a la guerra a defender su patria, tuvo que administrar el gane, lo que su país quería, en enfermería lo que el cliente precisa. Pero que poseía sentimientos al igual que el profesional de enfermería tiene un cúmulo de necesidades como profesional y como persona que debe desarrollar. Ulises quería volver a su esposa pero por tradición sabía que después de mucho tiempo ella debía estar casada al creerlo muerto .Encuentra a su regreso a su mujer asediada por pretendientes. El reconocimiento y la lucha vuelven a restituirle sus antiguos derechos. (LESKY, 1968, p. 61). Es la prudencia que debemos tener al considerar la situación del cliente y su familia. Y la "armonía conflictual " que experimenta un administrador del cuidado que no solo debe lidiar con el cliente sino también consigo mismo y con la institución.

Ulises representa la integración, que significa establecer una cultura de prevención de los cuidados (CROSBY, 1993, p. 11).

Para completar el hardware, lo que debe ser hecho tenemos el Cuidado que es definido como aquel fenómeno relacionado con el comportamiento de prestación de auxilio, apoyo o capacitación para un individuo o por otro individuo (o grupo ) con necesidades evidentes o anticipadas para mejorar o perfeccionar una condición o vida humana (GEORGE et al., 1993, p. 288). El cuidado está asociado a los conceptos de amor, preocupación, deber, intimidad y sexo (BOECHS \& PATRICIO, 1990, p. 114) y que se fundamenta en la concepción de salud.

Cuidado representado por Sancho Panza, el escudero de Quijote que tiene una visión más aterrizada pero entra en el "juego de cintura" de soñar e imaginar como vivir ese presente de la mejor manera. Su sueño es diferente, es lograr el poder, ser gobernador de una isla que le promete Don Quijote (trabajar con la heterogeneidad). Es concebir el propio mundo como un lugar de juegos como si se hubiese disuelto la diferencia entre jugar y lo que es serio (ZAJDSZNAJDER, 1992, p. 169).

En la segunda salida de Don Quijote para la aventura lo acompaña Sancho Panza con el asno. Sancho tácita o explícitamente, de modo voluntario y sin darse cuenta sigue a su señor, esto es, le remeda. Es la mejor figura de gracioso que ha creado el barroco español. Los dos tiempos de la burla, cuando se acoge al mundo ideal como si fuera real y al subrayar el choque de ambos, muestra su identidad con el caballero, pero también su diferencia tan importante de grado, por cuyo medio se consigue esa espléndida armonía (CASALDUERO, 1975, p. 29).

Surge entonces la contrapartida de las estrategias, el software, cómo debe ser hecho. Cómo hacer esa intervención con calidad? La respuesta cae en la Investigación constante que según el marco de referencia es el cuestionamiento sistemático, crítico y creativo con intervención competente en la realidad. Es pues una actitud cotidiana, no de hora marcada ni de lugar específico ni de instrumento especial (DEMO, 1994b, p. 34), es la base de una atención con calidad y de un accionar constante.

Representada por Quijote ese personaje creado por Miguel de Cervantes Saavedra que añora el pasado pero se despide de él para vivir el presente. Imagina todo lo que es de bueno de revivir del pasado para tolerar el presente. Cervantes expresa la idea de Destino, pero un destino histórico de una cultura que quiere mantener vivo el pasado. Pasado que el novelista ama, pero cuyo amor es fecundo porque lo ve, aunque con nostalgia 
irremediablemente muerto y por eso podrá integrarse sin reservas a salvar el presente (CASALDUERO, 1975, p. 23).

La determinación de la novela es el contraste entre la conducción, ocupación y medio social de un Hidalgo de la Mancha y el "más extraño pensamiento" que se le podía ocurrir: hacerse caballero andante, resucitar la Edad Media. Este extraño pensamiento se le ha originado leyendo libros de caballería (CASALDUERO, 1975, p. 24). Será necesario tener esta imaginación de Quijote para contrastar entre el ser y parecer, entre el idealismo y el realismo en el cuidado del cliente y poder avanzar en el presente.

La investigación tiene un poco de "imaginario" al proponerse preguntas norteadoras de lo que considera que puede ser porque así se dio en el pasado para llegar al presente que es lo que se vive en la condición de salud del niño.

Cómo se operacionaliza esa administración del cuidado? Con la educación que es la estrategia básica de formación humana, de aprender a aprender, saber pensar, para poder intervenir mejor e innovar (DEMO, 1994a, p. 21).

La educación implicará un proceso en el que la enfermera debe investigar con la familia del niño las medidas de poder y negociación para la prevención de la enfermedad, de los riesgos y conservar la vida saludable globalmente hablando. El niño, su familia y el profesional tienen una identidad cultural que les hace procurar información, formación para el desarrollo físico e intelectual.

Para que todos juntos puedan construir y participar de un mundo más saludable es preciso compartir los objetivos, deseos, modos de satisfacer esas metas, reestructurar los desafíos de su modo de vivir saludable y realizar los ajustes según las vivencias manifestadas y ocultas por el grupo familiar en su diario vivir.

Representada por Penélope, esposa de Ulises que organiza la artimaña de hacer un tejido que al ser concluido indicará su disponibilidad para casarse nuevamente. Y que deshace cada noche con la esperanza de que regrese su amado. Sin saber del regreso de Ulises se presenta ante los pretendientes en la sala, les anuncia la posibilidad de contraer nupcias y declara su propósito de que al día siguiente una competencia en el tiro al arco decidirá quién ha de ser su esposo. Ulises gana la competencia pero Penélope solo lo reconoce hasta que él le manifiesta un secreto relacionado al lecho nupcial (LESKY, 1968, p. $68)$.

La educación es ese fragmentar del cuidado que es individual según el cliente, la familia y lo vivido. Fragmentar es producir un pensamiento que no busca ligar o ligarse (ZAJDSZNAJDER, 1992, p. 26). Fragmentación que se da al deshacer el tejido según la vivencia de cada cliente, pero que se integra cada día cuando se reinicia la atención con miras a la prevención. Es la negociación de Penélope que enfrenta un cliente que no quiere ser conducido, quiere ser libre para hacer lo que quiere, no quiere un grupo misterioso planeando cada detalle de su vida (CROSBY; 1993, p. 6).

\section{MOSTRANDO EL CAMINO DE LA MEJORIA CONTINUA}

El Descongelamiento se dio en la etapa de la Visión donde se encontraron unidades de significado como vida, evolución, ejercer la ciudadanía, manifestación de sentimientos, creencias, expectativas y conquista de la autonomía. Veamos cómo se trabajaron esta unidades de significado en la Reestructuración, en la Implementación de las cuatro estrategias.

De hecho, administrar el cuidado según las expectativas, la forma de vivir la felicidad, la satisfacción implica respeto mutuo del cliente, sus padres y los profesionales de enfermería debido a la idiosincrasia de cada persona para enfrentar los acontecimientos de su vida. No se dio una discusión de lo esperado en el comportamiento de acuerdo a los acontecimientos vividos.

De parte del cliente externo la forma de manifestar los sentimientos, las creencias y las expectativas es sobreentendido pues aceptan que deben hablar poco sobre lo que sucede o piensan que ocurre. Consideran que los demás saben lo que es, incluso no preguntan si hay algo mejor.

También esperan pacientemente las indicaciones con relación a lo que deben hacerle a sus hijos, no preguntan, no hacen valer sus derechos. Así es difícil llevarlos a la conquista de su autonomía, a tener autodeterminación.

No se encontró ni en los padres ni en los clientes manifestación de sentimientos o de expectativas sobre lo que consideran que debe y puede ayudar la sociedad para mejorar la atención. No discuten sus derechos para tener la tecnología necesaria accesible para su beneficio, para tener el placer de vivir. Veámoslo en este caso:

Caso n.1 - Se trata de un niño con una leve ptosis parpebral izquierda por un probable hematoma epidural provocado por un accidente. Su madre no manifiesta los sentimientos de rabia o angustia por el accidente de su hijo. Parecía como si se tratara de un contratiempo que pasaría. El niño tenía dolor de cabeza pero ambos no lo decían, no pedian el analgésico, esperaban pacientemente a que la decisión partiese del profesional de enfermería. 
La conquista de la autonomía en la atención para la creación de un espacio saludable fue un aspecto discutido superficialmente e inclusive como actividad futura del hogar debido a una implícita ausencia de libertad para decidir el cambio en el ambiente durante el internamiento.

Se nota al igual que Ulises una prudencia en el actuar, a pesar de que consideraban que tenían los derechos para solicitar el cuidado. Se dio una armonía conflictual dentro del modelo biomédico, lo que no justifica que se deba continuar de la misma manera. Es el momento en que la institución y el empleado deberían ser bien sucedidos para que el cliente también lo sea. Hay un entrabamiento que se percibe como tensión en la atención, pero no es claro lo que hace difícil mostrar los puntos débiles y fuertes para la mejoría continua.

En la segunda estrategia, el Cuidado se encontró un total despreparo de los padres de los niños para vivir lo que hay que vivir bien o morir bien. Hay una lucha por la sobrevivencia así como el naufrago que desconoce donde se halla.

La evolución de la persona se da como una flecha que se lanza sin rumbo claro pero que fluye cumpliendo un principio físico que debe ser respetado aunque se desconozca su objetivo.

Muchas de las dificultades al conducir un proceso de evolución que se convierte en algo grotesco es debido a la falta de preparación, temor de hacer lo que puede ser hecho para progresar. Nadie puede parar el curso de la Naturaleza, pero si por imprudencia hacer las cosas más incómodas y lerdas y fastidiosas. Como un presente de la Naturaleza los niños que no son acompañados pueden evoluir y tener momentos de placer y felicidad que la cultura y la sociedad muchas veces mutila por medio de los adultos. Observémoslo en el caso a seguir.

Caso n. 2 - Un bello niño de once meses con diagnóstico de sepsis urinaria $y$ mielomeningocele operado es un ejemplo de evolución. Su sepsis fue ocasionada entre otros por malos cuidados de su familia, descuido en la ejecución del cateterismo intermitente. Cuando la conocí estaba acostada de lado, llorando con desespero por agarrar bien la chupeta que le tenían medio colocada. En la ejecución del cateterismo provocaba tristeza el observar la cantidad de secreción que se extraía. Qué cambio observamos? Conforme pasaron los días, al movilizarla, al darle comida, y al mejorar su sepsis urinaria, el niño reía, jugaba con sus manos, tomaba solo su chupeta, participaba del diálogo con otros niños y con el personal por medio de sonrisas, gestos guturales y miradas profundas.
La vida como un don precioso se defiende en todo tipo de relación y no es preciso inculcar esto en los padres ni en sus hijos, porque toda persona tiene una razón íntima indiscutible para defender su vida. Lo que las personas no comparten es la idea de que esa sobrevivencia pueda ser de buena u óptima calidad. Así en un momento de crisis de la familia no se puede recurrir a un pensamiento de sobrevivencia de buena calidad si no es su patrón de comportamiento.

El juego de cintura se da más en el profesional de enfermería que procura adaptar las medidas de cuidado, según el diagnóstico médico para preservar la vida. Los padres y los niños tienden a imaginar que la vivencia de la enfermedad es una crisis que debe pasar y procuran acomodarse a ella pero sin aceptarla plenamente. Es algo parecido a lo que hace Sancho con don Quijote, lo sigue, lo imita pero se contrapone.

En la tercera estrategia, la investigación encontramos que ejercer la ciudadanía como padres y hacerla efectiva para sus hijos requiere de un proceso largo y difícil pues desconocen los derechos del niño durante la hospitalización según la resolución n 41 da Declaración dos Direitos da Criança e do Adolescente (BRASIL, 1995).

Limité mi intervención a hacer preguntas que estimulasen su interés, pero la angustia por la sobrevivencia del niño y el respeto por las normas del hospital no ayudaban a criar un ambiente de cuestionamiento sistemático, crítico, creativo. Notemos la diferencia cuando se conocen los derechos.

Caso n. 3 - Sucedió algo especial con la madre de un jovencito con diagnóstico médico de tumor cerebral. Quizá por preocupación mía, debido a la desesperación de la madre con el dolor manifestado por su hijo y ante la respuesta inefectiva del personal, le di a leer la resolución $n 41$ (el artículo 7 que enfatiza el derecho del niño de no sentir dolor). A partir de ese momento la madre fue más vehemente en la presencia del médico hasta que este apareció, puncionó el cuero cabelludo, extrajo el líquido y se alivió el dolor.

La participación de los padres y de los clientes en el cambio de la sociedad y en hacer valer sus derechos como ciudadanos fue casi nula, pues más que comodismo, el momento de la enfermedad no permitía pensar qué era lo mejor para que se cambiara la atención de su situación de salud.

Se notó en los padres y en los niños una voluntad de vivir las experiencias del pasado como un secreto para soportar o integrarse al presente. Así como hacia Quijote entre lo que parece ser y lo que es irremediable. Sus 
pocas preguntas son para hacernos ver el abismo entre lo real y lo imaginario de sus pensamientos en relación a la enfermedad de los niños.

Con la cuarta estrategia, la Educación notamos que la forma de participación, de compartir el cliente y su familia en el diálogo, responde a veces a sentimientos diversos provocados por la situación de crisis. Las conductas son diversas, como diversas son las formas de reaccionar ante un mismo hecho o experiencia en la vida por diferentes personas de acuerdo con su cultura y experiencias pasadas.

La creatividad de los padres, del cliente y del profesional de enfermería para construir nuevas formas de atención de acuerdo con las circunstancias depende mucho de sus creencias, sentimientos, expectativas, de como ejercen su ciudadanía, de la conquista de la autonomía y de las perspectivas de vida que tienen. Mirémoslo en este caso.

Caso n. 4 - La niñita de tres meses tenía un mielomeningocele operado y problemas de insuficiencia respiratoria causados posiblemente por una hipertensión endocraneana no tratada después de una ventriculitis. El diálogo con los padres fue el día de salida de la niña del hospital. La creación de un ambiente saludable en otro hospital o en su hogar es imprescindible, ya que hubo una total separación de los padres con la evolución de la niña en su internamiento. La participación haciendo valer sus derechos según el artículo $n 1$ de la resolución $n 41$ (de protección de su salud sin discriminación por pocas probabilidades de vida) y el $n 13$ (de recibir todos los recursos terapeúticos disponibles como la colocación de una válvula para disminuirla hipertensión endocraneana) no fue ejecutada por los padres debido al desconocimiento de como vivir su ciudadanía y la de la niña.

El diálogo con los padres o los clientes para criar o hacer actividades de estimulación de potencialidades y capacidades del niño fue frustrado en algunos casos por ausencia de los mismos. En otros el respeto por su ciudadanía, sus derechos gritaban su único deseo de conservar la vida independientemente de las condiciones de su desarrollo, lo que anuló muchos esfuerzos en esta área.

En esta estrategia de la educación se ejerció la fragmentación de acuerdo a cada cliente. Así como Penélope, que no reconoce a Ulises, no hay claridad en el camino de la construcción y participación de los padres, los niños y los profesionales en el cuidado.

Un poco tratando de evaluar el propósito del inicio puedo decir que es durante la formación del profesional de enfermería que comienza la internalización de la necesidad de poseer un marco de referencia para ejecutar su trabajo diario. Los modelos pueden servir para formar, para orientar, pero no para normatizar, porque las personas aunque tengan un mismo diagnóstico médico, tienen una vivencia muy particular inclusive entre los que habitan en el mismo techo.

En el planeamiento se debe mirar para el ser humano como una persona global y a partir de esta visión ser aplicado en adultos, en niños hospitalizados, en la comunidad, en cualquier circunstancia de la vida.

Qué es necesario para planear? Es tener voluntad, espíritu de cambio, respeto por el otro, saber compartir, tener creatividad, en resumen deseos de investigar siempre, de no parar en el proceso, de tener ideas siempre y perseguirlas hasta su efectividad. La experiencia nos dice que esto no es una realidad en la práctica de enfermería. No es la cantidad de clientes la justificación para la elaboración del planeamiento de la atención y si el creer en la posibilidad de ejercitar la ciudadanía.

También existen dudas del lugar donde se debe registrar el plan. Es una cuestión ética importantísima. Debe ser un punto convergente de toda discusión de los profesionales de enfermería y de los clientes.

\section{CONSIDERACIONES FINALES}

Podemos concluir que la manifestación de las creencias, expectativas, sentimientos parece ser un tema olvidado en las relaciones dentro del modelo biomédico. El cuidado no es solicitado por el cliente o sus padres, no hay conquista de la autonomía.

La investigación tampoco es comprendida como actividad necesaria y eficaz en el trabajo diario del profesional de enfermería, menos aún como derecho de los padres para indicar lo que quieren.

La construcción y la participación con calidad en el cuidado de los clientes, padres y profesional de enfermería son difíciles ya que el diálogo es deficiente. Como consecuencia se torna difícil alcanzar una buena calidad de vida para que el niño pueda evoluir de acuerdo con los dictados de la naturaleza, teniendo muchas limitaciones en este caminar de la mejoría continua. Esto sucede casi siempre y no hacemos nada para modificar esta situación.

El ambiente donde enfermería funciona debe permitir el éxito con personas bien sucedidas. Esto implica conocer la cultura en la cual las personas se desarrollan para que podamos ayudarlas a conquistar la autonomía y consecuentemente la ciudadanía. No es fácil pero tampoco imposible. 


\section{THE IMPLEMENTATION IN THE OPERATIVE LEVEL OF THE NURSING PROCESS}

The development of the nursing care attention process guided through the PDCA. (plan, do, check, act), provides a continuous improvement of our actual context. The meaning of PDCA is translated as VIDA for: view, implementation, discernment, at just. For its application it takes in view the change theory of Kurt Lewin. This paper provides the results of the Implementation stage using different approaches as technical care, education, administration and research. These strategies show that people did not express their feelings, beliefs and their own expectations and autonomy in nursing care. They even do not develop their citizenship, right of living and being able to grow.

KEY WORDS: implementation, continuous improvement, autonomy, citizenship

\section{REFERENCIAS BIBLIOGRAFICAS}

01. ALBRECHT, K.; BRADFORD, L.J. Serviços com qualidade: a vantagem competitiva. São Paulo: Makron Books, 1992.

02. BOEHS, A.E.; PATRÍCIO, Z.M. O qué é cuidar/ cuidado. Rev Esc Enfermagem USP, São Paulo, v. 24, n. 1, p. 111-116, abril 1990.

03. BRASIL. Ministério da Justiça. Resolução n. 41 de outubro de 1995: Direitos da criança e do adolescente hospitalizados. Brasilia: Ministério da Justiça, 1995.

04. CASALDUERO, J. Sentido y forma del Quijote. Madrid: Insula, 1975.

05. CERQUEIRA, E.P.de. Paradigmas da qualidade. Rio de Janeiro: Imagem, 1992.

06. G Gestão da qualidade: princípios e métodos. São Paulo: Pioneira, 1993.

07. CORRÊEA, P.A.M. Qualidade total, da visão à sistematização. Rio de Janeiro: Imagem, 1993.

08. CROSBY, P.B. Integração: qualidade e recursos humanos para o ano 2000. São Paulo: Makron Books, 1993.
09. DEMO, P. Educacão e qualidade. Campinas: Papirus, 1994a.

10. Pesquisa e construção de conhecimento: metodología científica no caminho de Habermas. Rio de Janeiro: Tempo Brasileiro, 1994b.

11. GEORGE, B.J. et al. Teorías de enfermagem. Porto Alegre: Artes Médicas, 1993.

12. LEDDY, S.; PEPPER, J.M. Bases conceptuales de la enfermería profesional. Filadelfia: J. B. Lippincott, 1985.

13. LESKY, A. Historia de la literatura griega. Madrid: Gredos, 1968.

14. LEWIN, K. Field theory in social science. Greenwood Press, 1951.

15. MALIK, A.M. Desenvolvimiento de recursos humanos, gerência de qualidade e cultura das organizações de saúde. Rev.Admin.Empresas, São Paulo, v. 32, n. 4, p. 32-41, 1992.

16. ZAJDSZNAJDER, L. A travessía do pósmoderno: nos tempos do vale -tudo. Rio de Janeiro: Gryphus, 1992. 